\title{
A Machine-Assisted Gaze Analysis Method for Students' Psychological Evaluation
}

\author{
Huiling Wang $\mathbb{D}^{1}$ and Yafei Shan $\mathbb{D}^{2}$ \\ ${ }^{1}$ School of Education Science, Henan Vocational University of Science and Technology, Zhoukou 466000, China \\ ${ }^{2}$ Student Affairs Department, Zhoukou Normal University, Zhoukou 466001, China \\ Correspondence should be addressed to Yafei Shan; 20182028@zknu.edu.cn
}

Received 6 January 2022; Revised 26 January 2022; Accepted 5 February 2022; Published 21 February 2022

Academic Editor: Wen Zeng

Copyright (c) 2022 Huiling Wang and Yafei Shan. This is an open access article distributed under the Creative Commons Attribution License, which permits unrestricted use, distribution, and reproduction in any medium, provided the original work is properly cited.

\begin{abstract}
This article combines machine intelligence-assisted gaze analysis to construct a student psychological evaluation system, which provides a basis for solving student psychological evaluation methods. At the same time, by recognizing students' behavioral characteristics and then their psychological characteristics, a PC-side application with multiple functions such as humancomputer interaction, user data management, and physiological data display and storage has been developed. In addition, this article constructs an intelligent system structure to evaluate students' psychology through expert diagnosis. Finally, this article evaluates the students' psychology through the system and at the same time uses the comparative test method to evaluate the system. Experimental research shows that the student psychological evaluation system based on machine intelligence-assisted gaze analysis proposed in this paper is very close to the expert diagnosis result of student psychological evaluation.
\end{abstract}

\section{Introduction}

Educational evaluation can be classified according to different criteria. According to the content of the evaluation, it is divided into condition evaluation, process evaluation, and result evaluation. Moreover, according to the evaluation criteria, it is divided into relative evaluation, absolute evaluation, and intraindividual difference evaluation. According to the subjects participating in the assessment, it is divided into self-assessment and others' assessment. In addition, according to the time and role of evaluation, it is divided into diagnostic evaluation, formative evaluation, and summative evaluation. Finally, according to the method of evaluation, it is divided into quantitative evaluation and qualitative evaluation. Most scholars divide social work evaluation into two basic types: formative evaluation and cumulative evaluation. The former refers to those methods that provide information about project activities [1]. The provision of this type of information can theoretically help the organization develop and improve the project. The latter is to examine the situation in which the project accomplishes its goals. This type of information is very useful for funding agencies to decide whether to reopen the project or continue to allocate funds to the project. The initial cumulative evaluation may later be transformed into a formative goal, which is used to help the project improve, rather than to summarize the results achieved or no results. The other two concepts are process evaluation and result evaluation. Among them, the process evaluation occurs in the project in the operation stage, and the result evaluation involves the project's results to the client and the community [2].

College students are the successors of the country's future career development, and they are at a critical transition stage from adolescence to adulthood. At this time, their outlook on life, world outlook, and values gradually stabilized, and at the same time, they are in a relatively critical transitional stage. At this stage, college students grow up quickly and start a relatively independent life. Interpersonal relationships with parents, teachers, and peers need to be handled properly, and they are in a stage of high incidence of psychological conflicts. On the one hand, college students are required to have a healthy body, and on the other hand, 
they also need to have a good psychological quality to face the pressures of all aspects of society. This makes paying attention to the mental health of college students an indispensable link in higher education [3].

At present, the methods of mental quality assessment include homework method, projection method, and questionnaire method. The homework method is to allow the testee to perform the actual operation, and then, the tester will make a qualitative psychological quality assessment based on his performance in the homework. This method can solve the problem of subject evaluation due to educational and cultural factors, but this method depends on the cognitive level of the tester, and the evaluation results of different testers are quite different and too subjective. The projection method is to respond to ambiguous images, ink stains, or sentences. Subjects are asked to answer freely according to their own understanding and feelings and to understand the subject's internal language and psychological characteristics. This method is the same as the homework method and is not fixed and accurate. Based on the evaluation basis, the individual's psychological quality cannot be accurately evaluated.

This article combines machine intelligence-assisted gaze analysis to construct a student psychological evaluation system and conducts research on the psychological evaluation of college students to improve the effect of college student psychological evaluation.

\section{Related Work}

The standard of mental health is an important basis for evaluating mental health, and it is an evaluative index for connotation and definition. At the same time, mental health evaluation standards are mostly defined around the concept and connotation of mental health [4]. Literature [5] regards mental health as a mental state in terms of emotion, intelligence, and social adaptation, including emotional stability, keen wisdom, and good social adaptation. The mental health standards in the literature [6] are emotional health, personality health, good social adaptation, and harmonious interpersonal relationships. Literature [7] puts forward 6 criteria for mental health: striving for self-growth, treating oneself objectively, unity of outlook on life, establishing harmonious interpersonal relationships, acquiring knowledge and skills necessary for life, and loving life and having empathy. The 6 criteria of mental health proposed in the literature [8] are as follows: positive self-evaluation, moderate control and sense of dominance, realistic optimism, ability to care for others, ability to be happy and satisfying, and ability to be enthusiastic about constructive and creative work. Literature [9] believes that mental health includes six abilities, which are self-knowledge, self-realization, personality integration, autonomy, reality testing, and environmental control. The literature [10] believes that mentally healthy people often have 7 characteristics, which are generally reasonable, have a sense of self-growth, have the ability to love, have good intimacy, adapt to reality, work well, and have the ability to have a better life. Literature [11] puts forward 10 criteria for mental health: adequate self-safety, understand- ing of one's own abilities, goals are in line with reality, not divorced from society, healthy personality, able to learn from experience, good interpersonal relationships, control one's emotions, give full play to one's own personality, and meet personal needs. Literature [12] believes that a mentally healthy person, that is, a mature person, should possess 15 abilities and characteristics including self-sense, independence, dependence, adaptation, reality testing, the ability to love and be loved, and the ability to control emotions. The literature [13] summarized the evaluation criteria of mental health into four aspects: self-knowledge and self-attitude, interpersonal attitude and social skills, enthusiasm for life and the ability to solve problems effectively, and the internal coordination of personality structure. Literature [14] proposes that the standards of mental health include love of work, good interpersonal relationships, understanding of oneself, and acceptance of the external environment. Literature [15] believes that mentally healthy people should pay attention to self-exploration and discovery, improve selfvalues and self-esteem, have ideals closely integrated with reality, and be down-to-earth. Literature [16] proposes that three criteria can be used as the basis for judging mental health. One is statistical criteria, which compares individual behavior scores with the average state of the numerical distribution of normal behavior; the other is sociological criteria, which compares individual. The behavior is compared with social norms; the third is medical standards, whether there are mental illnesses. Based on the above standards, it is not difficult to find that the formulation of mental health standards is an extremely comprehensive and complex issue. Combining different standards at home and abroad, the author sums up three levels of mental health standards: first, the physical and mental level, including physical health, no mental illness, able to fully understand self, balance internal psychological conflicts, good at learning and sharing, and having a healthy personality; the second is the level of interpersonal relationships, with good interpersonal interaction, positively accepting others, and being able to get a sense of happiness in communication; the third is at the social level, with good social adaptability, able to actively participate in work, and realize their own value. Comprehensive consideration of these aspects will help to clarify many problems of mental health, so as to play a certain enlightenment role for follow-up related research.

After the mathematical model is established, certain results can be derived through logical reasoning or mathematical operations. If a certain explanation is given to the model, the result can be regarded as a certain prediction of the empirical system [17]. The predicted value is further compared with the actual test value, and the mathematical model can be revised according to the degree of agreement between the two. The advantage of using mathematical models to describe psychological phenomena is not only that it has greater generality, accuracy, deductive power, and predictive power than natural language descriptions, but more importantly, it is convenient for computer simulations and creates for the development of artificial intelligence. Condition: psychological statistics is a branch of applied statistics that studies how to collect, sort, and analyze digital data in 


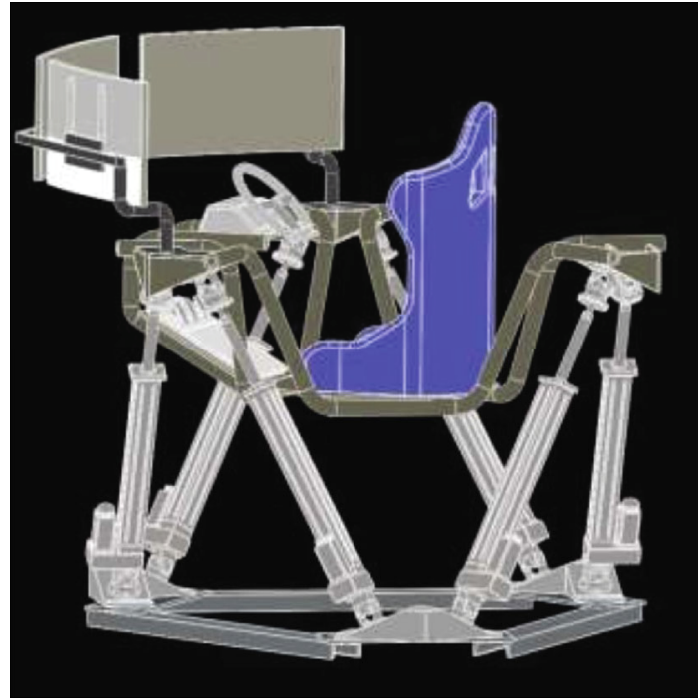

Figure 1: Three-dimensional model of intelligent auxiliary gaze analysis platform.

psychological experiments or investigations and how to make scientific inferences based on the information conveyed by these data [18]. Psychological statistics is one of the effective tools for psychological research. The history of the development of psychology proves that scientific psychology is inseparable from scientific experiments or investigations, and psychological experiments or investigations must face the problem of processing digital data. For example: how to collect data to make the numbers most meaningful and reflect the research topics; what methods are used to organize and analyze the data to maximize the information reflected by these data, so as to make a scientific analysis of the results of experiments or investigations. Explain: how can we infer from the partial results obtained to the overall, make general scientific conclusions, and so on. To solve these problems, we must rely on scientific statistical methods [19].

\section{Machine Intelligence-Assisted Gaze Analysis}

The prototype of the machine intelligence auxiliary gaze analysis platform used in this topic is shown in Figure 1. The main intelligent auxiliary platform is composed of a fixed platform, a motion platform, and six motion branches of the same UPU. The 6 UPU branch chains are used as the drive input of the mechanism, and the length of each branch is changed by the expansion and contraction of the electric cylinder to control the pose of the platform. Both the fixed platform and the moving platform are connected to the two ends of each electric cylinder through a Hooke hinge, thus forming a six-degree-of-freedom platform.

The degree of freedom of the intelligent auxiliary platform is calculated:

$$
F=6(n-g-1)+\sum_{i=1}^{g} f_{i}
$$

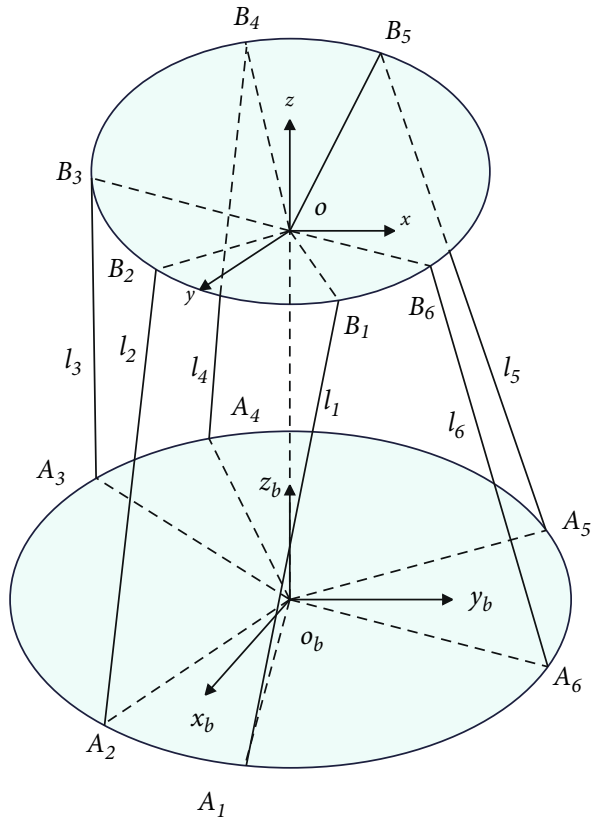

FIGURE 2: The coordinate system of the intelligent auxiliary platform.

TABLE 1: The actual values of the geometric parameters of the prototype (unit: $\mathrm{mm}$ ).

\begin{tabular}{lccc}
\hline$R_{1}$ & $R_{2}$ & $h$ & $h_{1}$ \\
\hline 800.0 & 800.0 & 1000.0 & 380.0 \\
\hline
\end{tabular}

In the formula, $n$ is the number of components, $g$ is the number of motion pairs, and $f_{i}$ is the number of degrees of freedom of each motion pair, $i=1,2, \cdots, n$. It can be seen from Figure 1 that the total number of components of the platform is $n=14$, and the number of kinematic pairs is $g$ $=18$, where each drive branch chain kinematic joint is composed of Hooke hinges $(f=2)$ and moving joints $(f=1)$. Substituting it into formula (1), the degree of freedom of the platform can be calculated as 6 .

In order to describe the pose of the intelligent auxiliary platform, this paper establishes a spatial rectangular coordinate system, including a fixed coordinate system $O_{b}-x_{b} y_{b} z_{b}$ and a moving coordinate system $O-x y z$, as shown in Figure 2. Among them, the lower platform is a fixed platform, the upper platform is a moving platform, the Hooke hinge joints of the branch chain connected with the fixed platform and the moving platform are, respectively, denoted as $A_{i}, B_{i}($ $i=1,2, \cdots, 6)$, and the driving branch chain is denoted by $f$. The two coordinate systems are described below [20].

(1) Inertial coordinate system $O_{b}-x_{b} y_{b} z_{b}$ (denoted as $P$ ): the coordinate origin $O_{b}$ is located at the geometric center of the fixed platform, the $x_{b}$ axis is perpendicular to the line $A_{1} A_{6}$, the $z_{b}$ axis is perpendicular to the plane determined by the hinge point $A_{1}, A_{2} \cdots$, $A_{6}$, and the $y_{b}$ axis can be determined by the righthand rule 


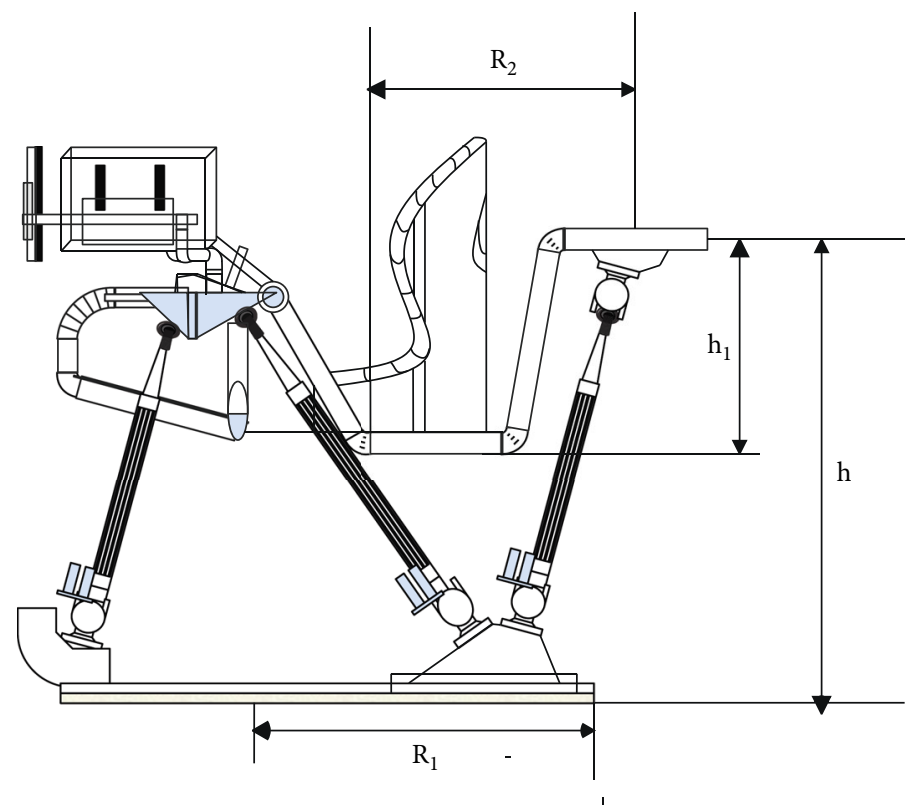

FIGURE 3: Schematic diagram of platform geometric parameters.

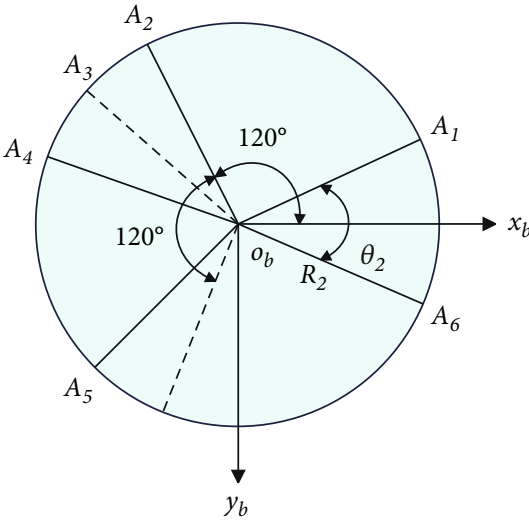

(a)

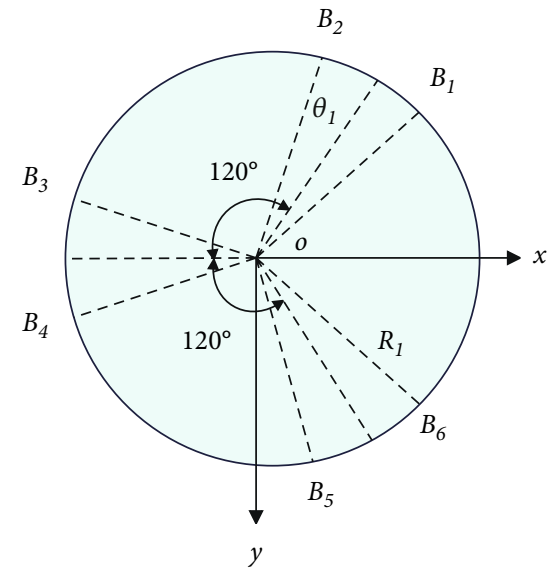

(b)

FIgure 4: Plan view of coordinate system. (a) Coordinate diagram of the fixed platform plan. (b) Coordinate diagram of the dynamic platform plan.

(2) The moving coordinate system, that is, the upper platform body coordinate system $O-x y z$ (denoted as $M$ ): the coordinate origin $O$ is located at $h_{1}$ below the circle where $B_{1}, B_{2}, \cdots, B_{6}$ is located, and this position is the geometric center of the upper platform, the $x$-axis is perpendicular to the line $B_{1} B_{6}$, the $y$-axis is perpendicular to the plane determined by $B_{1}, B_{2}, \cdots, B_{6}$ and the plane is straight up, and the $z$-axis is determined by the right-hand rule

The geometric parameters of the platform structure are shown in Table 1. Among them, $R_{1}$ is the radius of the fixed platform, $R_{2}$ is the radius of the circle (circle $O_{2}^{\prime}$ ) where $B_{1}, B_{2}, \cdots, B_{6}$ is located, $h$ is the distance between the center of the fixed platform and the center of the motion platform, $h_{1}$ is the distance between the circle $O_{2}^{\prime}$ and the geometric center of the motion platform, and the geometric meaning of each parameter can be seen in Figure 3.

It can be seen from Figure 3 that there is a vector equation for each branch.

$$
\mathbf{A}_{i} \mathbf{B}_{i}=\mathbf{O}_{1} \mathbf{O}_{2}+\mathbf{O}_{2} \mathbf{B}_{i}-\mathbf{O}_{1} \mathbf{A}_{i} \quad(i=1,2, \cdots, 6) .
$$

$A_{i}$ in the fixed coordinate system $O_{b}-x_{b} y_{b} z_{b}$ can be expressed as

$$
A_{i}=\left[R_{1} \cos \eta_{i} R_{1} \sin \eta_{i} 0\right]^{\mathrm{T}}(i=1,2, \cdots, 6) .
$$

$B_{i}$ in the moving coordinate system $O-x y z$ can be expressed as 


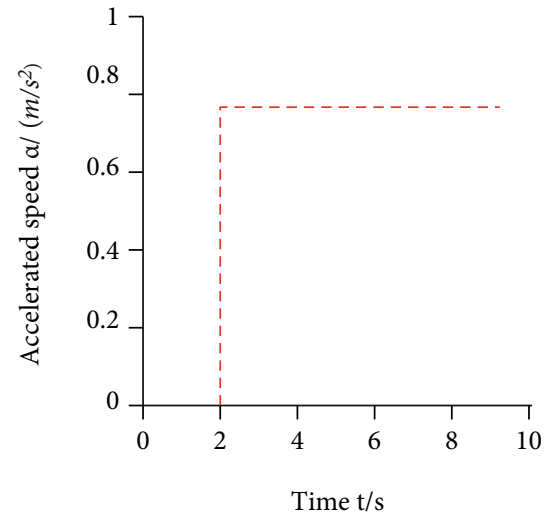

(a)

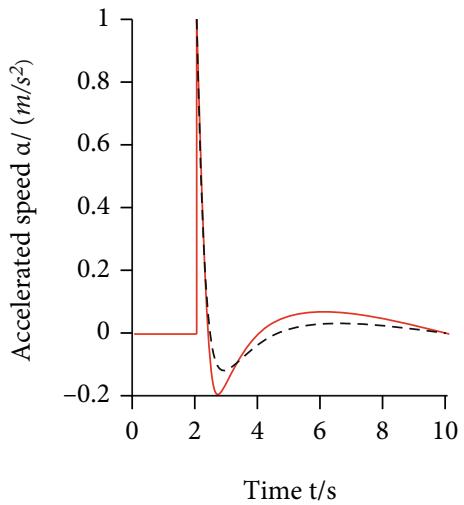

- - - Second order — Third order

(b)

Figure 5: Acceleration signal output. (a) Acceleration input signal. (b) Export acceleration of the high-pass filter.

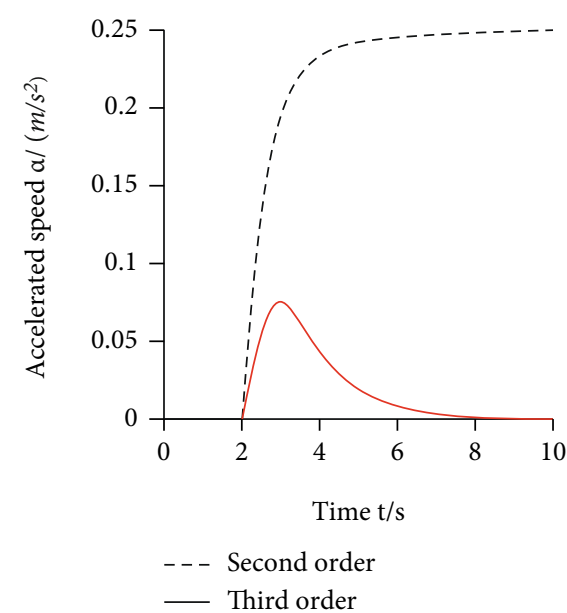

FIgURE 6: Output displacement of the high-pass filter.

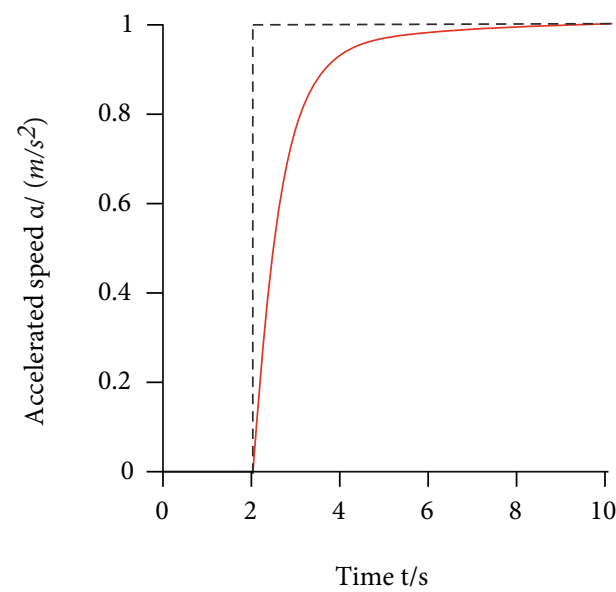

- - - Second order

— Third order

FIgURE 7: Comparison of input and output of low-pass filter.

$$
B_{i}=\left[\begin{array}{lll}
R_{2} \cos \xi_{i} & R_{2} \sin \xi_{i} & 0
\end{array}\right]^{\mathrm{T}}(i=1,2, \cdots, 6) .
$$

In the formula, $\eta_{i}$ and $\xi_{i}$ are the polar coordinate angles of the plane where each hinge point is located.

$O_{b}-x_{b} y_{b} z_{b}$ and $O-x y z$ are projected into the plane determined by the hinge point, and the plane coordinate system is established, as shown in Figures 4(a) and 4(b).

In the plane coordinate system, $\eta_{i}$ and $\xi_{i}$ are expressed as

$$
\begin{aligned}
& \left\{\begin{array}{l}
\eta_{1}=\frac{\theta_{2}}{2}, \eta_{2}=\frac{2 \pi}{3}-\frac{\theta_{2}}{2}, \eta_{3}=\frac{2 \pi}{3}+\frac{\theta_{2}}{2}, \\
\eta_{4}=\frac{4 \pi}{3}-\frac{\theta_{2}}{2}, \eta_{5}=\frac{4 \pi}{3}+\frac{\theta_{2}}{2}, \eta_{6}=-\frac{\theta_{2}}{2},
\end{array}\right. \\
& \left\{\begin{array}{l}
\xi_{1}=\frac{\pi}{3}-\frac{\theta_{1}}{2}, \xi_{2}=\frac{\pi}{3}+\frac{\theta_{1}}{2}, \xi_{3}=\pi-\frac{\theta_{1}}{2}, \\
\xi_{4}=\pi+\frac{\theta_{1}}{2}, \xi_{5}=-\frac{\pi}{3}-\frac{\theta_{1}}{2}, \xi_{6}=-\frac{\pi}{3}+\frac{\theta_{1}}{2} .
\end{array}\right.
\end{aligned}
$$

When analyzing the movement of the platform, Euler angles are generally used to describe the attitude of the moving platform in the inertial coordinate system. Initially, the initial orientation of the moving coordinate system $M$ is parallel to the inertial coordinate system $P$. First, the algorithm turns $O-x y z$ around its $z$-axis by angle $a$ to obtain $O-$ $x^{(1)} y^{(2)} z$ (denoted as $M^{(1)}$ ) and then rotates around $M^{(1)}$ 's $y^{(1)}$-axis by angle $\beta$ to obtain $O-x^{(2)} y^{(1)} z^{(2)}$ (denoted as $\left.M^{(2)}\right)$, and finally, the algorithm rotates $x^{(2)}$ around $M^{(2)}$ by angle $\gamma$ to get $O-x^{(2)} y^{(3)} z^{(2)}$ (denoted as $M^{(3)}$ ). Each rotation can be represented by a rotation coefficient matrix, and the above rotation process can be represented as

$$
R=\left[\begin{array}{ccc}
c \alpha c \beta & c \alpha s \beta s \gamma-s \alpha c \gamma & c \alpha s \beta c \gamma+s \alpha s \gamma \\
s \alpha c \beta & s \alpha s \beta s \gamma+c \alpha c \gamma & s \alpha s \beta s \gamma-c \alpha s \gamma \\
-s \beta & c \beta s \gamma & c \beta c \gamma
\end{array}\right]
$$




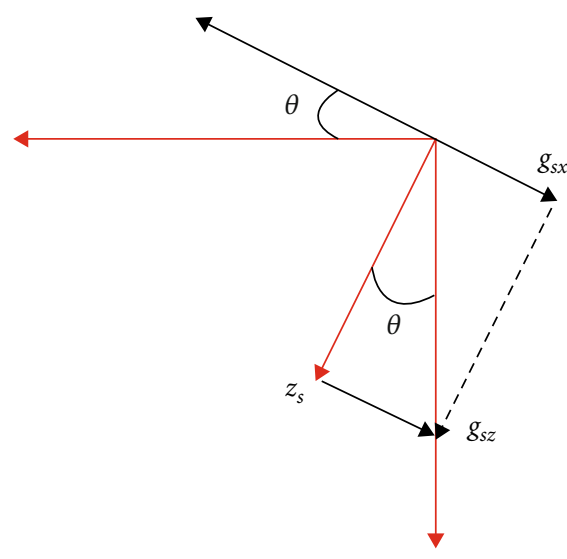

FIGURE 8: Tilt coordination.

In the formula, $c \alpha=\cos \alpha, s \alpha=\sin \alpha$ and others can be deduced by analogy.

The rotation transformation matrix has the following properties:

$$
\left\{\begin{array}{l}
\mathbf{R}^{T}=\mathbf{E} \\
\mathbf{R}^{T}=\mathbf{R}^{-1}
\end{array}\right.
$$

In the formula, $\mathbf{E}$ is the identity matrix.

The position of $\mathbf{O}_{b} \mathbf{B}_{i}$ in the inertial coordinate system $\mathrm{O}_{b}-x y z$ can be expressed as

$$
\mathbf{O}_{b} \mathbf{B}_{i}=\mathbf{R}_{3}+\mathbf{R} \mathbf{B}_{i}^{\prime}(i=1,2, \cdots, 6) .
$$

In the formula, $\mathbf{R}_{3}$ is the position vector of the origin $O$ of the dynamic coordinate system relative to $O_{b}$, that is, $\mathbf{R}_{3}$ $=\mathbf{O}_{b} \mathbf{O}=\left[\begin{array}{lll}x & y & z\end{array}\right]^{\mathrm{T}}$. If it is assumed that each member does not undergo elastic deformation, the inverse solution equation for the drive branch is as follows:

$$
\mathbf{l}_{i}=\mathbf{R}_{3}+\mathbf{R} \mathbf{B}_{i}^{\prime}-\mathbf{O}_{b} \mathbf{A}_{i}(i=1,2, \cdots, 6) .
$$

From formula (9), the inverse kinematic solution of the mechanism can be expressed as

$$
l_{i}=\left|A_{i} B_{i}\right|=\sqrt{\left\|R_{3}+R B_{i}^{\prime}-O_{b} A_{i}\right\|^{2}}(i=1,2, \cdots, 6) .
$$

In the formula, $l_{i}$ is the length of the drive branch $A_{i} B_{i}$.

The classic washout algorithm includes comparison calculation, proportional link, coordinate transformation link, acceleration high-pass filter link, acceleration lowpass filter link, angular velocity high-pass filter link, tilt coordination link, angular velocity limiting link, and various integration links. This section will design the main links.

Because students feel the movement of the real intelligent auxiliary platform through the vestibular system of the head, in order to simulate motion, it is necessary to convert the acceleration at the center of mass of the intelligent auxiliary platform into the force of the student's head. It is necessary to calculate the specific force in the coordinate system of the intelligent auxiliary platform as

$$
f_{\mathrm{T}}=a_{\mathrm{T}}-g_{\mathrm{T}} .
$$

In the formula, $a_{\mathrm{T}}$ is the acceleration $n$ of the real intelligent auxiliary platform in the intelligent auxiliary platform coordinate system $\left(\mathrm{m} / \mathrm{s}^{2}\right) ; g_{\mathrm{T}}$ is the acceleration of gravity ( $\mathrm{m} / \mathrm{s}^{2}$ ) in the intelligent auxiliary platform coordinate system, and $g_{\mathrm{T}}=-g \cdot[\sin \theta, \cos \theta \sin \varphi, \cos \theta \cos \varphi]^{\mathrm{T}}, \theta$ and $\varphi$ are the pitch and roll angles of the intelligent auxiliary platform, respectively.

Taking into account the limited range of motion of the intelligent auxiliary platform, a proportional link (also known as signal reduction link) is added to the washout algorithm to prevent excessive acceleration or angular velocity signals from causing the platform to exceed its range of motion. At present, the most widely used method is the cubic polynomial reduction method, and its general expression is

$$
y=-0.001 x^{3}-0.03 x^{2}+x
$$

Since the washout filtering algorithm filters the specific force and angular velocity signals in the inertial coordinate system, it is necessary to perform coordinate transformation on the specific force signal and angular velocity signal of the student's head calculated in the intelligent auxiliary platform coordinate system. It is converted into a signal in the inertial coordinate system, and the process can be realized byformulas (13) and (15).

$$
f_{2}=\mathbf{L}_{s} f_{1} .
$$

Among them, $f_{1}$ is the specific force in the coordinate system of the intelligent auxiliary platform $\left(\mathrm{m} / \mathrm{s}^{2}\right) ; f_{2}$ is the specific force in the inertial coordinate system $\left(\mathrm{m} / \mathrm{s}^{2}\right) ; \mathbf{L}_{s}$ is the coordinate transformation matrix, and

$$
\mathbf{L}_{s}=\left[\begin{array}{ccc}
c \theta c \psi & s \varphi s \theta c \psi-c \varphi s \psi & c \varphi s \theta c \psi+s \varphi s \psi \\
c \theta s \psi & s \varphi s \theta s \psi+c \varphi c \psi & c \varphi s \theta s \psi-s \varphi c \psi \\
-s \theta & s \varphi c \theta & c \varphi c \theta
\end{array}\right] .
$$

In the formula, $s \varphi=\sin \varphi, c \varphi=\cos \varphi$ and others can be deduced by analogy.

$$
\omega_{2}=T_{s} \omega_{1} .
$$

Among them, $\omega_{1}$ is the specific force in the coordinate system of the intelligent auxiliary platform $(\mathrm{rad} / \mathrm{s}) ; \omega_{2}$ is the specific force in the inertial coordinate system $(\mathrm{rad} / \mathrm{s})$; $\mathbf{T}_{s}$ is the coordinate transformation matrix, and

$$
\mathbf{T}_{s}=\left[\begin{array}{ccc}
1 & \sin \varphi \tan \theta & \cos \varphi \tan \theta \\
0 & \cos \varphi & -\sin \varphi \\
0 & \sin \varphi \sec \theta & \cos \varphi \sec \theta
\end{array}\right] .
$$




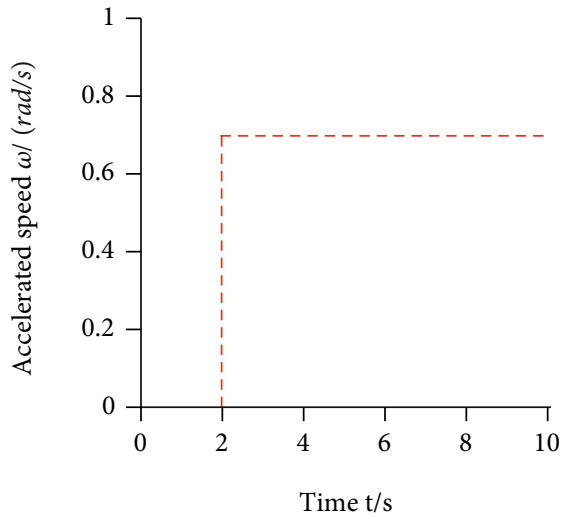

(a)

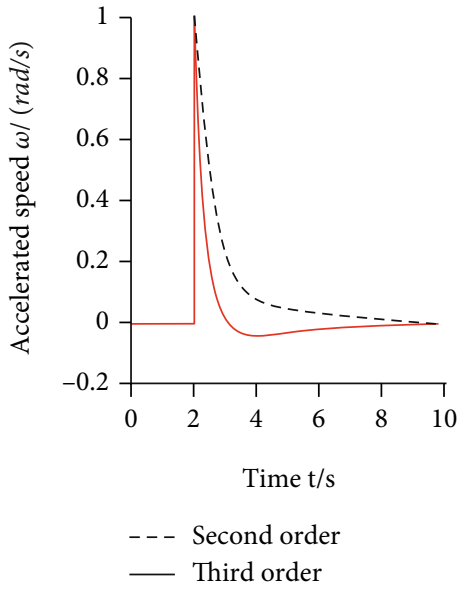

(b)

FIGURE 9: Angular velocity signal output. (a) Angular velocity input signal diagram. (b) Comparison of output angular velocity of high-pass filter.

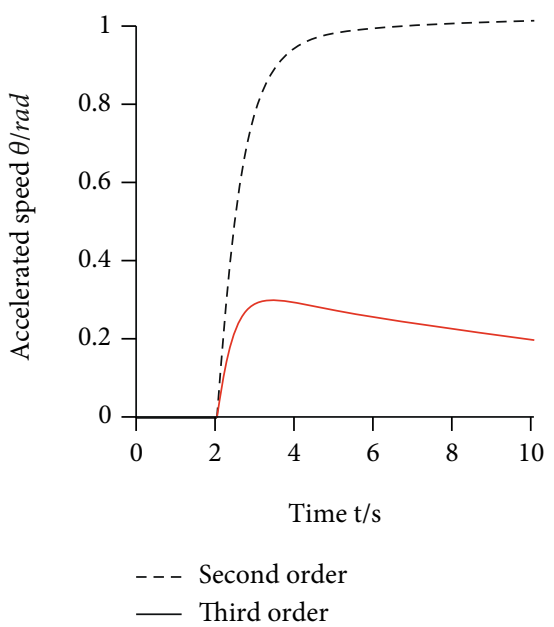

Figure 10: Comparison of output angles of high-pass filters.

In order to simulate sudden acceleration and ensure that the platform does not exceed its range of motion, the acceleration high-pass filter is used to filter out the low-frequency signals in the acceleration signal and retain its highfrequency components. The general form of the high-pass filter equation is

$$
\frac{Y}{X}=\frac{s^{n}}{D(s)}
$$

In the formula, $D(s)=\sum_{i=0}^{n} a_{i} s^{i}, a_{0} \neq 0$.

When simulating a certain continuous acceleration, for example, the input is a unit step signal, and after a long enough time, the output displacement is

$$
x_{\text {final }}=\lim _{t \longrightarrow \infty} x=\lim _{s \longrightarrow 0} s\left\{\frac{1}{s}\left[\frac{s^{n}}{D(s)} \frac{1}{s^{2}}\right]\right\}=\lim _{s \longrightarrow 0} \frac{s^{n-2}}{D(s)}
$$

In order to ensure that the platform can return to the neutral position after simulating a certain sudden movement, that is, the high-frequency part of the acceleration, $x_{\text {find }}=0$ should be made. When $n \geq 3$, the lowest order of the high-pass filter is 3. At this time, the transfer function is

$$
H(s)=\frac{s^{3}}{\left(s^{2}+2 \xi_{h} \omega_{h} s+\omega_{h}^{2}\right)\left(s+\omega_{m}\right)} .
$$

In the formula, $\omega_{h}$ is the second-order natural response frequency $(\mathrm{rad} / \mathrm{s}) ; \omega_{m}$ is the first-order natural response frequency of the inertial link $(\mathrm{rad} / \mathrm{s}) ; \xi_{h}$ is the second-order damping ratio.

In order to test the features of the acceleration highpass filter, the initial input is 0 , and a unit step signal is given at time, as shown in Figure 5(a). This signal is input into the second-order and third-order acceleration highpass filters, respectively, and the filtered acceleration can be obtained, as shown in Figure 5(b). The displacement can be obtained after the acceleration is integrated twice, as shown in Figure 6.

It can be seen from Figure 5(b) that the acceleration output of the two acceleration filters is not much different, and high-frequency acceleration can be obtained. However, it can be found from Figure 6 that the displacements output by the two acceleration filters are significantly different. After a long enough time, the displacement output by the second-order acceleration filter is a nonzero constant value, that is, the platform does not return to the neutral position. In this way, the platform does not have enough space for movement to simulate the next sudden movement. The displacement output by the third-order acceleration filter is finally 0 , and the platform will have a sufficient range of motion to simulate the next sudden movement.

Therefore, the acceleration high-pass filters along the $x, y$, and $z$ directions all adopt a third-order structure, as shown in 


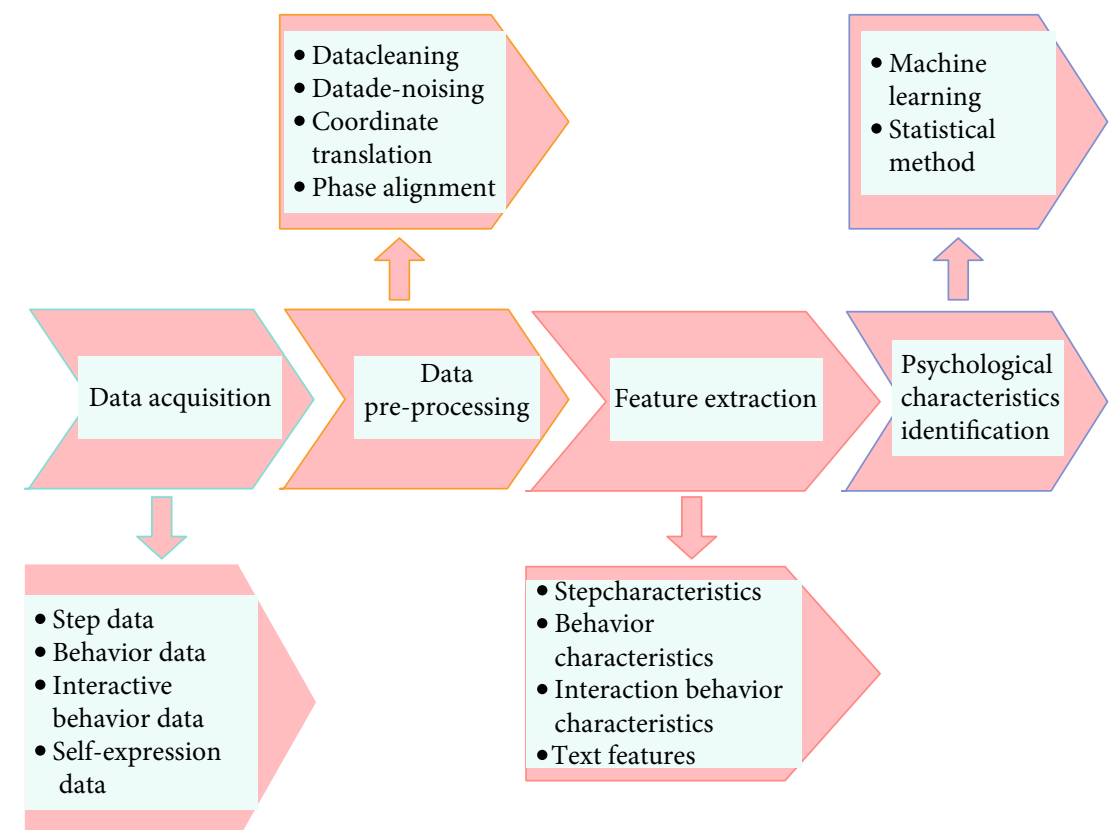

FIGURE 11: The evaluation process of students' psychological features based on machine intelligence-assisted gaze analysis.

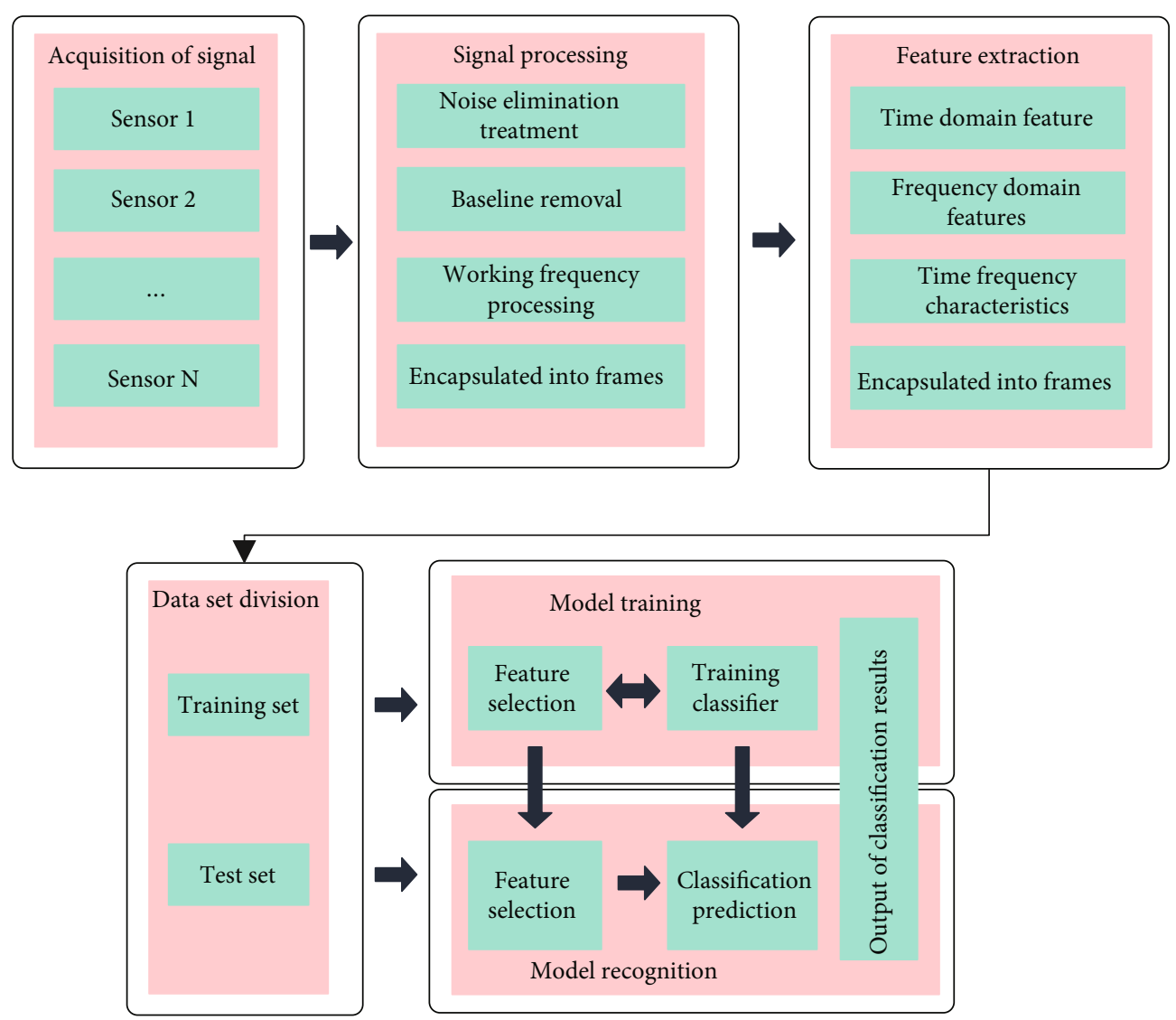

Figure 12: Psychological stress evaluation. 

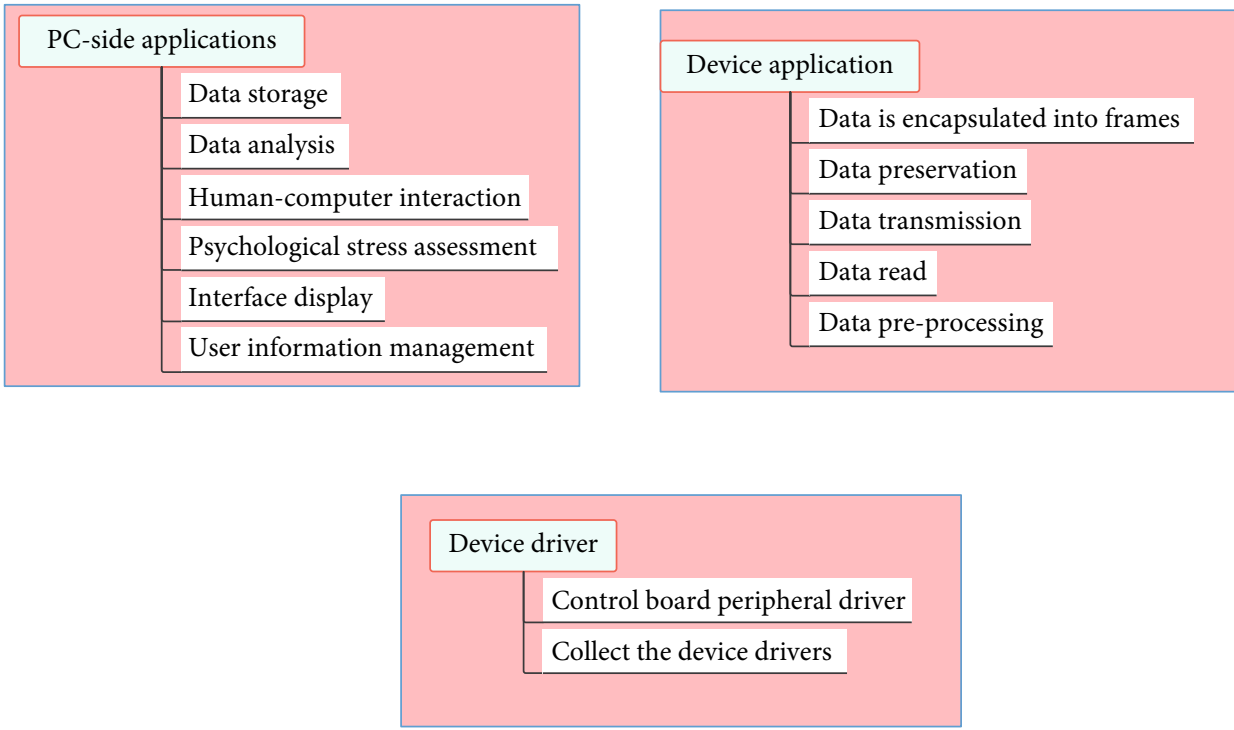

Figure 13: The overall software architecture of the system.

$$
\left\{\begin{array}{l}
H_{x}(s)=\frac{s^{3}}{\left(s^{2}+2 \xi_{h x} \omega_{h x} s+\omega_{h x}^{2}\right)\left(s+\omega_{m x}\right)}, \\
H_{y}(s)=\frac{s^{3}}{\left(s^{2}+2 \xi_{h y} \omega_{h y} s+\omega_{h y}^{2}\right)\left(s+\omega_{m y}\right)}, \\
H_{z}(z)=\frac{s^{3}}{\left(s^{2}+2 \xi_{h z} \omega_{h z} s+\omega_{h z}^{2}\right)\left(s+\omega_{m z}\right)} .
\end{array}\right.
$$

In order to simulate continuous acceleration, that is, the low-frequency component in the acceleration, the platform needs to be tilted to a certain angle, and the gravity component is used to simulate the continuous acceleration felt by human otoliths. For the vertical force signal, there is no low-pass filter in this direction because the low-frequency component cannot be simulated through tilt coordination.

The low-pass acceleration channel uses a second-order low-pass filter, and the transfer function is

$$
\left\{\begin{array}{l}
H_{x}(s)=\frac{\omega_{l x}^{2}}{s^{2}+2 \xi_{l x} \omega_{l x} s+\omega_{l x}^{2}}, \\
H_{y}(s)=\frac{\omega_{l y}^{2}}{s^{2}+2 \xi_{l y} \omega_{l y} s+\omega_{l y}^{2}} .
\end{array}\right.
$$

In the formula, $\omega_{l x}, \omega_{l y}$ is the natural cutoff frequency of the second-order low-pass filter $(\mathrm{rad} / \mathrm{s}) ; \xi_{l x}$, $\xi_{l y}$ is the damping ratio.

In order to test the features of the acceleration lowpass filter, the initial input is 0 , a unit step signal is given at $2 \mathrm{~s}$, and the signal is input to the low-pass filter to obtain the filtered acceleration, as shown in Figure 7 . It can be found from Figure 7 that the low-frequency component of acceleration can be obtained through the second-order low-pass filter.
When the simulation platform rotates an angle, a continuous forward or backward specific force can be obtained in the corresponding direction, and the specific force is generated by the gravity component. Since the human body cannot distinguish whether the specific force is generated by acceleration or the gravity component, the specific force can simulate continuous acceleration. As shown in Figure 8, the positive $x$ direction is the forward direction of the simulation platform, which is consistent with the $>$ forward direction of the real intelligent auxiliary platform. The continuous specific force $g \sin \theta$ can be obtained by rotating (pitching) the simulation platform around the $y$-axis. The magnitude of the specific force is related to the tilt angle.

The Euler angle for continuous acceleration is [21]

$$
\left\{\begin{array}{l}
\theta_{\mathrm{t}}=\arcsin \left(\frac{f_{l x}}{g}\right), \\
\varphi_{\mathrm{t}}=-\arcsin \left(\frac{f_{l y}}{g \cos \theta}\right) .
\end{array}\right.
$$

In general, the pitch and roll angles produced by tilt coordination are very small, so formula (22) can be written in the form of

$$
\left\{\begin{array}{l}
\theta_{\mathrm{t}}=\frac{f_{l x}}{g} \\
\varphi_{\mathrm{t}}=-\frac{f_{l y}}{g}
\end{array}\right.
$$

When simulating continuous acceleration, the angular velocity of the simulated platform tilt should be less than the human sensory threshold in the previous section to ensure that the process is not noticed by the students, and the purpose of increasing the angular velocity limit link 
TABLE 2: Comparison of the results of the student psychological evaluation system based on machine intelligence-assisted gaze and expert diagnosis.

\begin{tabular}{lccccc}
\hline Number & $\begin{array}{c}\text { Expert } \\
\text { diagnosis }\end{array}$ & $\begin{array}{c}\text { System } \\
\text { diagnosis }\end{array}$ & Number & $\begin{array}{c}\text { Expert } \\
\text { diagnosis }\end{array}$ & $\begin{array}{c}\text { System } \\
\text { diagnosis }\end{array}$ \\
\hline 1 & 94.97 & 94.28 & 31 & 76.22 & 75.39 \\
2 & 94.90 & 95.34 & 32 & 76.04 & 78.91 \\
3 & 94.75 & 93.92 & 33 & 75.22 & 72.25 \\
4 & 93.96 & 96.73 & 34 & 74.56 & 72.66 \\
5 & 92.89 & 89.99 & 35 & 73.34 & 76.70 \\
6 & 92.40 & 96.84 & 36 & 72.90 & 76.30 \\
7 & 90.77 & 94.82 & 37 & 72.27 & 70.62 \\
8 & 90.53 & 93.71 & 38 & 71.95 & 73.74 \\
9 & 90.10 & 86.45 & 39 & 71.66 & 75.19 \\
10 & 89.82 & 90.48 & 40 & 71.52 & 73.47 \\
11 & 88.75 & 85.78 & 41 & 71.36 & 74.68 \\
12 & 88.23 & 90.67 & 42 & 70.99 & 70.02 \\
13 & 87.81 & 83.99 & 43 & 70.88 & 73.13 \\
14 & 87.56 & 86.00 & 44 & 70.29 & 72.28 \\
15 & 86.43 & 87.21 & 45 & 69.53 & 68.02 \\
16 & 85.82 & 82.01 & 46 & 69.32 & 66.14 \\
17 & 84.31 & 83.84 & 47 & 69.21 & 70.87 \\
18 & 83.74 & 86.15 & 48 & 68.44 & 69.65 \\
19 & 82.56 & 84.11 & 49 & 66.85 & 69.20 \\
20 & 82.55 & 82.50 & 50 & 64.09 & 64.99 \\
21 & 80.76 & 84.00 & 51 & 63.53 & 61.18 \\
22 & 79.82 & 83.56 & 52 & 63.39 & 60.57 \\
23 & 79.48 & 76.28 & 53 & 62.85 & 60.72 \\
24 & 79.32 & 76.13 & 54 & 62.70 & 62.27 \\
25 & 78.43 & 79.20 & 55 & 62.02 & 59.15 \\
26 & 78.05 & 78.69 & 56 & 61.14 & 63.71 \\
27 & 77.84 & 81.33 & 57 & 59.37 & 59.71 \\
28 & 77.74 & 77.57 & 58 & 59.12 & 60.37 \\
29 & 77.13 & 80.01 & 59 & 58.57 & 58.29 \\
\hline 0 & 76.50 & 79.92 & 60 & 58.43 & 56.02 \\
\hline & & & & &
\end{tabular}

comes from this. In addition, tilt coordination relies on a certain angle of tilt to generate gravity components to simulate continuous acceleration. Under normal circumstances, the acceleration of the intelligent auxiliary platform is limited, the corresponding tilt angle is not large, the vertical component of gravity will not decrease too much, and the students will not easily feel the attenuation of the vertical component of gravity. In general, the continuous acceleration simulated by the tilt coordination method cannot exceed $4 \mathrm{~m} / \mathrm{s}^{2}$, so the maximum tilt coordination angle cannot exceed $25^{\circ}$.

In order to simulate the situation of sudden angular velocity and to ensure that the platform does not exceed its range of motion, the angular velocity high-pass filter is used to filter out the low-frequency signal in the angular velocity signal and retain its high-frequency components. From the previous section, we know that the general form of the high-pass filter equation is given by equation (17).

When simulating a certain continuous angular velocity, for example, the input is a unit step signal, and after a long enough time, the output angle is

$$
\alpha_{\text {find }}=\lim _{t \longrightarrow \infty} \alpha=\lim _{s \longrightarrow 0}\left\{\frac{1}{s}\left[\frac{s^{n}}{D(s)} \frac{1}{s}\right]\right\}=\lim _{s \longrightarrow 0} \frac{s^{n-1}}{D(s)}
$$

In order to ensure that after simulating a certain sudden movement, that is, the high-frequency part of the angular velocity, the half station can return to the neutral position, $\alpha_{\text {final }}=0$ should be made. When $n \geq 2$, the lowest order of the high-pass filter is 2 . At this time, the transfer function is [22]

$$
H(s)=\frac{s^{2}}{s^{2}+2 \xi_{h} \omega_{h} s+\omega_{h}^{2}} .
$$

In the formula, $\omega_{h}$ is the second-order natural response frequency $(\mathrm{rad} / \mathrm{s}) ; \xi_{h}$ is the second-order damping ratio.

In order to test the features of the angular velocity high-pass filter, the initial input is 0 , and a unit step signal is given at $2 \mathrm{~s}$, as shown in Figure 9(a). The signal is input into the first-order and second-order angular velocity high-pass filters, respectively, and the filtered angular velocity can be obtained, as shown in Figure 9(b). The angle can be obtained by integrating the angular velocity once, as shown in Figure 10.

It can be seen from Figure 9(b) that the angular velocities output by the two angular velocity filters are not much different, and high-frequency angular velocities can be obtained. However, it can be found from Figure 10 that the output angles of the two angular velocity filters are significantly different. After a long enough time, the output angle of the first-order angular velocity filter is a nonzero constant. In other words, the platform did not return to the median. In this way, the movement range of the platform to simulate the next sudden movement situation will be limited. Moreover, the angle output by the second-order angular velocity filter will eventually be 0 , and the platform will have a sufficient range of motion to simulate the next sudden movement.

Therefore, the angular velocity high-pass filters around the $x, y$, and $z$ directions all adopt a second-order structure, as shown in

$$
\left\{\begin{array}{l}
H_{x}(s)=\frac{s^{2}}{s^{2}+2 \xi_{h x} \omega_{h x} s+\omega_{h x}^{2}}, \\
H_{y}(s)=\frac{s^{2}}{s^{2}+2 \xi_{h y} \omega_{h y} s+\omega_{h y}^{2}}, \\
H_{z}(s)=\frac{s^{2}}{s^{2}+2 \xi_{h z} \omega_{h z} s+\omega_{h z}^{2}} .
\end{array}\right.
$$




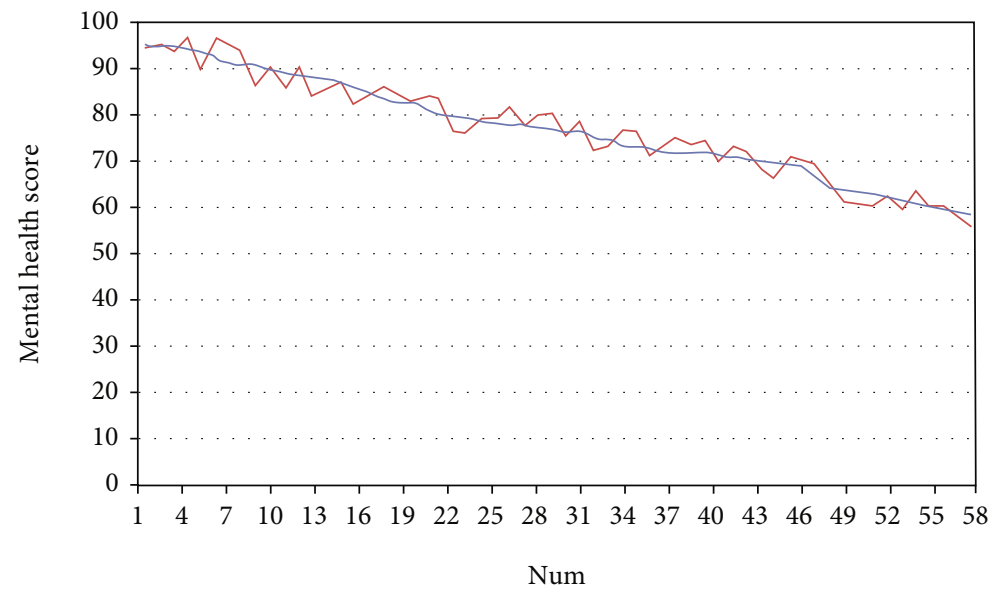

Figure 14: Effect analysis of student psychological evaluation system based on machine intelligence-assisted gaze.

\section{Psychological Evaluation of Students Based on Machine Intelligence-Assisted Gaze Analysis}

The above constructs a student psychological evaluation system based on machine intelligence-assisted gaze. The system mainly recognizes students' behavior features and uses behavior features to recognize students' psychological features. Figure 11 shows the psychological feature evaluation process.

Psychological stress, also known as psychological stress, is the physical and psychological state of physical and mental tension that the human body is stimulated by internal and external environments. Considering that the characteristics of physiological signals cannot be concealed, the psychological stress state of the human body can be objectively and effectively evaluated through the physiological signals of the human body. The psychological stress assessment method of this system is shown in Figure 12, including physiological data collection, preprocessing, feature extraction, data set division, training process, evaluation process, and three types of psychological stress assessment outputs: calm, mild stress, and high pressure.

This system not only completes the design of the software and hardware of the physiological signal acquisition system but also develops a PC-side application with multiple functions such as human-computer interaction, user data management, and physiological data display and storage. The entire software part consists of three parts: device driver, device application, and PC-side application. Figure 13 is the overall software architecture of the system.

Based on the above research, this paper evaluates the student psychological evaluation system based on machine intelligence-assisted gaze built in this paper. This article evaluates students' psychology through expert diagnosis and uses the obtained standards as basic data. After that, the students' psychology is evaluated uniformly through the system of this paper, and the two sets of data are compared, and the results shown in Table 2 and Figure 14 are obtained.
From the above analysis, it can be seen that the evaluation results of student psychological evaluation system based on machine intelligence-assisted gaze proposed in this paper are relatively close to the expert diagnosis results, and the system constructed in this paper can be applied to student psychological evaluation in the future.

\section{Conclusion}

The most commonly used scale questionnaire method for student psychological evaluation uses structured questions, which allows subjects to answer with "yes" or "no" or a limited number of choices, and scores judgments based on the answer results. The scale method is mostly a questionnaire formulated according to a specific test purpose, and at the same time, a conventional model must be established as a basis for judgment. In view of the shortcomings of the commonly used methods of psychological quality assessment in the past, especially the absolute status of the evaluation of human factors, many psychologists have devoted themselves to studying how to more objectively and accurately assess the individual's psychological quality. In order to explore a reliable method of student psychology evaluation, this paper combines machine intelligence-assisted gaze analysis to analyze student psychology. Through experimental research, it can be known that the evaluation results of the student psychological evaluation system based on machine intelligenceassisted gaze proposed in this paper are relatively close to the expert diagnosis result.

\section{Data Availability}

The data used to support the findings of this study are available from the corresponding author upon request.

\section{Conflicts of Interest}

The authors declare that they have no known competing financial interests or personal relationships that could have appeared to influence the work reported in this paper. 


\section{Acknowledgments}

This work was supported by the School of Education Science, Henan Vocational University of Science and Technology.

\section{References}

[1] J. Park, U. G. Kang, and Y. Lee, "Big data decision analysis of stress on adolescent mental health," Journal of The Korea Society of Computer and Information, vol. 22, no. 11, pp. 89-96, 2017.

[2] J. Deckro, T. Phillips, A. Davis, A. T. Hehr, and S. Ochylski, "Big data in the veterans health administration: a nursing informatics perspective," Journal of Nursing Scholarship, vol. 53, no. 3, pp. 288-295, 2021.

[3] F. F. Nastro, D. Croce, S. Schmidt, R. Basili, and F. SchultzeLutter, "Insideout project: using big data and machine learning for prevention in psychiatry," European Psychiatry, vol. 64, no. S1, pp. S343-S343, 2021.

[4] H. Jung and K. Chung, "Social mining-based clustering process for big-data integration," Journal of Ambient Intelligence and Humanized Computing, vol. 12, no. 1, pp. 589-600, 2021.

[5] M. Gonçalves-Pinho, J. P. Ribeiro, and A. Freitas, "Schizophrenia related hospitalizations-a big data analysis of a national hospitalization database," Psychiatric Quarterly, vol. 92, no. 1, pp. 239-248, 2021.

[6] M. Gonçalves-Pinho, J. P. Ribeiro, A. Freitas, and P. Mota, "The use of big data in psychiatry-the role of pharmacy registries," European Psychiatry, vol. 64, no. S1, pp. S793-S793, 2021.

[7] M. V. Rudorfer, "Psychopharmacology in the age of "big data": the promises and limitations of electronic prescription records," CNS Drugs, vol. 31, no. 5, pp. 417-419, 2017.

[8] A. B. Shatte, D. M. Hutchinson, and S. J. Teague, "Machine learning in mental health: a scoping review of methods and applications," Psychological Medicine, vol. 49, no. 9, pp. 1426-1448, 2019.

[9] W. N. Price and I. G. Cohen, "Privacy in the age of medical big data," Nature Medicine, vol. 25, no. 1, pp. 37-43, 2019.

[10] J. Liu, X. Zhai, and X. Liao, "Bibliometric analysis on cardiovascular disease treated by traditional Chinese medicines based on big data," International Journal of Parallel, Emergent and Distributed Systems, vol. 35, no. 3, pp. 323-339, 2020.

[11] D. Wilfling, A. Hinz, and J. Steinhäuser, "Big data analysis techniques to address polypharmacy in patients-a scoping review," BMC Family Practice, vol. 21, no. 1, pp. 1-7, 2020.

[12] Y. Wang, L. Kung, W. Y. C. Wang, and C. G. Cegielski, “An integrated big data analytics-enabled transformation model: application to health care," Information \& Management, vol. 55, no. 1, pp. 64-79, 2018.

[13] A. Hong, B. Kim, and M. Widener, "Noise and the city: leveraging crowdsourced big data to examine the spatiotemporal relationship between urban development and noise annoyance," Environment and Planning B: Urban Analytics and City Science, vol. 47, no. 7, pp. 1201-1218, 2020.

[14] X. Cheng, L. Fang, X. Hong, and L. Yang, "Exploiting mobile big data: sources, features, and applications," IEEE Network, vol. 31, no. 1, pp. 72-79, 2017.

[15] M. Moessner, J. Feldhege, M. Wolf, and S. Bauer, "Analyzing big data in social media: text and network analyses of an eating disorder forum," International Journal of Eating Disorders, vol. 51, no. 7, pp. 656-667, 2018.

[16] J. Miller, R. Atala, D. Sarangarm et al., "Methamphetamine abuse trends in psychiatric emergency services: a retrospective analysis using big data," Community Mental Health Journal, vol. 56, no. 5, pp. 959-962, 2020.

[17] M. Gonçalves-Pinho, J. P. Ribeiro, and A. Freitas, "Schizophrenia hospitalizations-a big data approach," European Psychiatry, vol. 64, no. S1, pp. S157-S158, 2021.

[18] R. T. Perdue, J. Hawdon, and K. M. Thames, "Can big data predict the rise of novel drug abuse?," Journal of Drug Issues, vol. 48, no. 4, pp. 508-518, 2018.

[19] S. Graham, C. Depp, E. E. Lee et al., "Artificial intelligence for mental health and mental illnesses: an overview," Current Psychiatry Reports, vol. 21, no. 11, pp. 1-18, 2019.

[20] J. Popham, J. Lavoie, and N. Coomber, "Constructing a public narrative of regulations for big data and analytics: results from a community-driven discussion," Social Science Computer Review, vol. 38, no. 1, pp. 75-90, 2020.

[21] P. Balthazar, P. Harri, A. Prater, and N. M. Safdar, "Protecting your patients' interests in the era of big data, artificial intelligence, and predictive analytics," Journal of the American College of Radiology, vol. 15, no. 3, pp. 580-586, 2018.

[22] A. Stellbrink and E. Meisenzahl, "Big data market analysis of ehealth in medical neuroscience," European Psychiatry, vol. 41, no. S1, pp. S39-S39, 2017. 